CLINICAL CASE

\title{
LAPAROSCOPIC INTERVENTION IN HIRSCHSPRUNG DISEASE - MARTIN DUHAMEL METHOD
}

\author{
Maria Pușcașu ${ }^{1}$, Lavinia Iftene ${ }^{1}$, D.N. Păduraru ${ }^{1,2}$ \\ 'The University of Medicine and Pharmacy "Carol Davila", Bucharest, Romania \\ ${ }^{2}$ General Surgery and Emergency Clinic III - The University Emergency Hospital of Bucharest, Romania \\ Corresponding author: Dan Nicolae Păduraru \\ Phone no. 0040744756443 \\ E-mail: dan.paduraru.nicolae@gmail.com
}

\begin{abstract}
Hirschsprung's disease or congenital megacolon is a developmental disorder characterized by the absence of nerve cells in the myenteric plexus in the distal colon. The specific symptoms are caused by the absence of the peristaltic movement in the affected segment. $75 \%$ of cases involve the rectosigmoid region, and $60 \%$ of children affected have other associated congenital diseases (ophthalmological, urogenital, cardiac or central nervous system anomalies). We are presenting the case of a newborn delivered at term who develops symptoms represented by: abdominal distension, delay in the pass of meconium, food intolerance and vomiting all indicating the clinical picture of low intestinal obstruction. Following clinical examination, abdominal radiographs, contrast enema, serial biopsies of rectal and colonic wall as well as anorectal manometry the diagnosis of congenital megacolon - Hirschsprung disease is placed. The article includes a detailed description of the surgical technique used in this case: Duhamel-Martin Laparoscopic assisted method. Evolution is favorable, with no early or long term complications. The laparoscopic assisted Duhamel technique is among the first therapeutic options when it comes to addressing a newborn with Hirschsprung's disease, a technique not commonly used but with very good prognosis in the presented case.
\end{abstract}

Keywords: Hirschprung disease, Martin Duhamel Method

\section{Introduction}

Hirschsprung's disease or congenital megacolon is a developmental disorder characterized by the absence of nerve cells in the myenteric plexus in the distal colon. The functional obstruction occurs due to the absent peristalsis in the affected segment. Hirschsprung disease affects only the recto-sigmoid region in $75 \%$ of cases. About $60 \%$ of children with Hirschsprung's disease show other disorders of variable severity. $43 \%$ of children experience eye disorders, 20\% show congenital abnormalities of the genitourinary tract, 5\% have congenital heart disease, $5 \%$ suffer hearing impairments, and $2 \%$ show central nervous system abnormalities. Hirschsprung disease is associated with chromosomal abnormalities or syndromes in approximately $9 \%$ of cases [1]. Incidence is found to be the one case in 5000 newborns and a boy in 4. Most cases of Hirschsprung's disease are diagnosed in the 
neonatal period, when the passing of meconium does not occur in the first $24-48 \mathrm{~h}$ of life. While contrast enema or anorectal manometry are useful diagnostic methods, rectal and colonic wall biopsy is the standard criterion. Once the diagnosis is confirmed, surgical treatment consists of removing the aganglionic bowel as well as to manage the continuity of the healthy bowel with the distal rectum.

\section{The case presentation}

We report the case of a newborn male, J.A., delivered at term who within 48 hours postapartum presented: abdominal distension, delay in passing meconium, food intolerance and bilious vomiting; clinical picture suggestive for low intestinal obstruction.

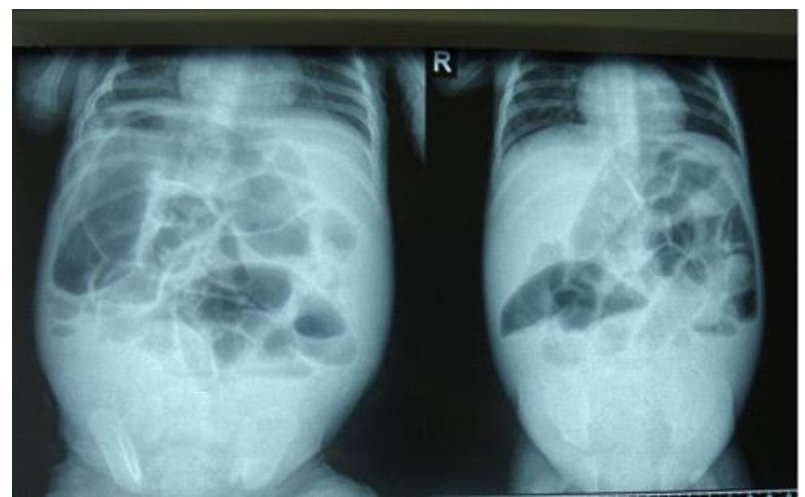

Figure 1 - Abdominal Radiography - Frontal and Posterior View

Following abdominal radiographs, contrast enema, serial biopsies of rectal and colonic wall as well as anorectal manometry the diagnosis of congenital megacolon - Hirschsprung disease is placed

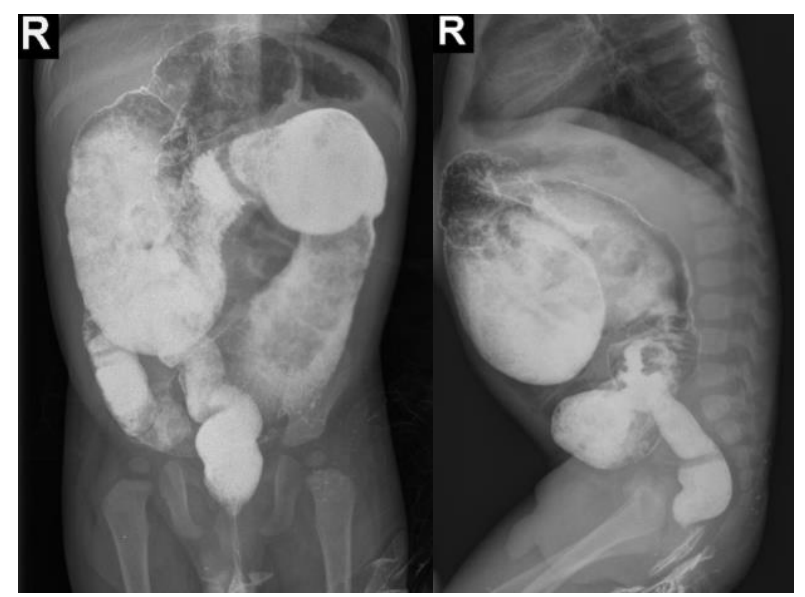

Figure 2. Abdominal Radiography - Frontal and Lateral View

\section{Technique}

The surgical therapeutic option in this case has to be the laparoscopically assisted Duhamel Method which actually represents the abdominoperineal retrorectal intrasfincterian descending of the proper colon in order to reestablish the continuity of the digestive tract through anastomoses [2]. The key steps of the procedure are laparoscopic exploration, rectal mobilization, colon dissection, the pull-through manoeuvre as well as the two final anastomoses: a terminolateral one and a laterolateral one. Throughout the next paragraphs we will be presenting the operating technique used as in the present pacient's case. The surgical evolution as well as the postoperatory evolution remained favorable throughout the entire procedure and the patient was discharged 6 days after the operation.

\section{Surgical steps}

1. Laparoscopic and biopsical exploration to determine the transition zone of the disease by serial biopsies of the colonic wall, or by suction of the rectal mucosa.

2. After receiving the extemporaneous histological biopsy results, the laparoscopic dissection of the rectum is proceeded.

3. Laparoscopic dissection of the sigmoid colon

4. Transection of the rectum at the peritoneal reflection either internally after ligature of the proximal rectum, or after transanal mobilization.

5. Transanal opening of the distal posterior rectum, development of the retrorectal space from below, and retrorectal pull-through of colon till ganglionated colon becomes visible.

\section{Anastomosis.}

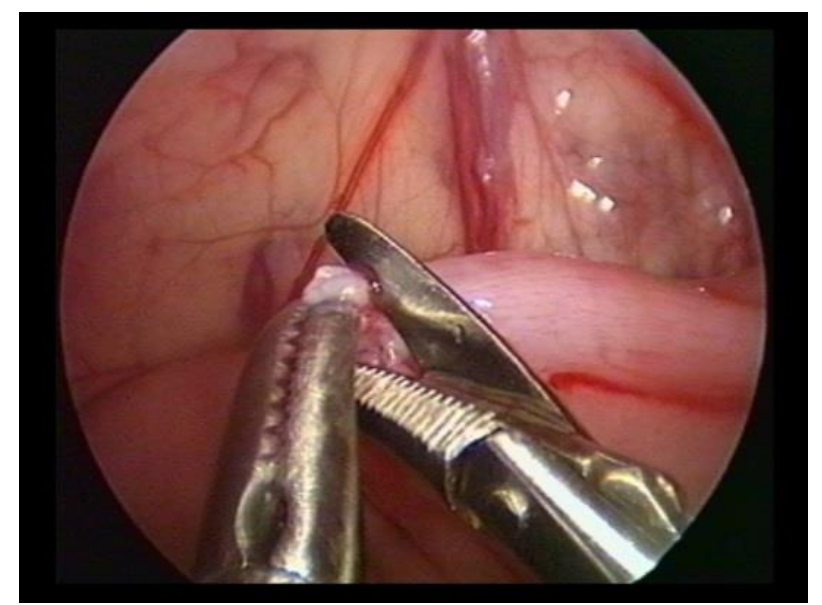

Figure 3a - Laparoscopical Biopsy 


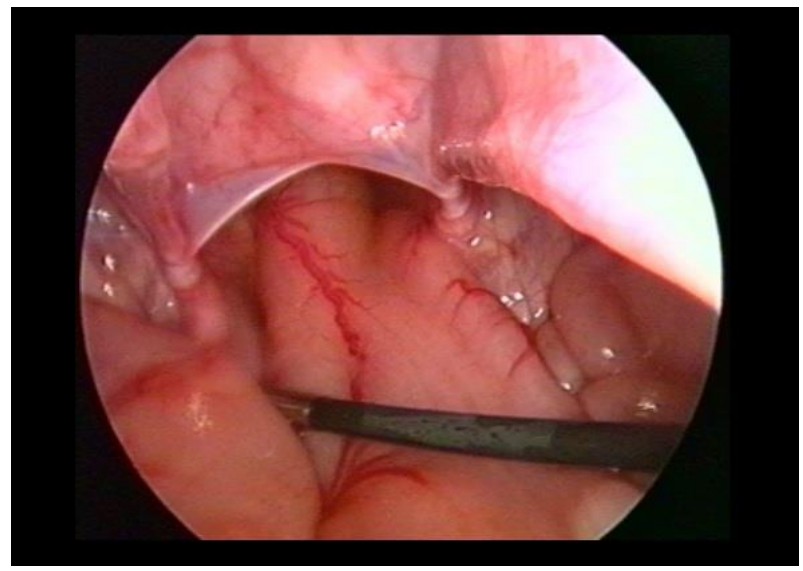

Figure 3b - Laparoscopical Biopsy

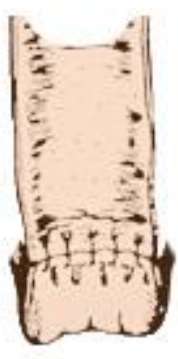

a

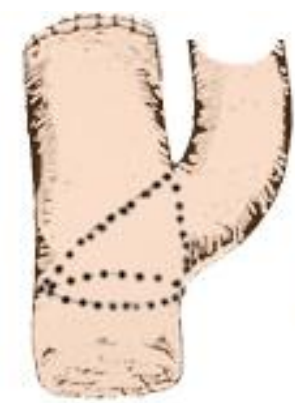

b

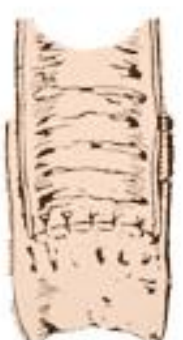

c
Figure 4 - Classical Hirschsprung Disease Surgical Procedures - Swenson (a), Duhamel(b), Soave(c)

\section{Method}

While several techniques (Swenson, Soave) [3] are in use for rectosigmoid disease , the Duhamel-type technique is prefered for extended aganglionosis. Generally, an initiative preoperative session for extracting biopsies is undergone either laparoscopically or through rectal wall suction, transanally.

This depends on the timing of the diagnosis and on the effectiveness of bowel decompression achieved by rectal washouts. For infants diagnosed within the neonatal period, the operation should be performed between the age of 1 and 4 months [4].

Preoperative preparation: The day before surgery, an antegrade whole bowel lavage is carried out.

Positioning of the patient: Older children are placed in a supine position at the end of the operating table. Small children can be placed transversely at the end of the operating table.

Considering Anesthesia: - General anesthesia is used in combination with locoregional techniques. Tracheal intubation is commonly preffered. The rectum is emptied with a large double-lumen suction device. A urine catheter is inserted after draping. The bladder is emptied regularly during the operation by the surgeon.

\section{Operative steps}

1. Minimal subombilical incision for introduction of the Veres needle and the first trocar. Pneumoperitoneum is firstly obtained and afterwards the first trocar is „blindly” introduced.

2. The next 3 trocars are inserted in the infant's abdomen. A suction cannula which can also be used for manipulation of the rectum during dissection is placed in the rectum.

3. The sigmoid colon is being retracted cephalad. The rectal dissection is started from the right. A window is created in the mesentery at the rectosigmoid junction close to the bowel wall, the dissection is continued anteriorly, and the peritoneal reflection is divided. The rectum is mobilized circumferentially, and stops just inferior to the peritoneal reflection. Next, the dissection is continued caudad, close to the posterior wall of the rectum, and ends just superior to the anus.

4. The dissection now proceeds cephalad. If the upper sigmoid colon has to be mobilized, Toldt's line is incised first, to mobilize the mesentery. The dissection of the sigmoid colon mesentery is carried out close to the colon wall until the normal ganglionic colon, as confirmed by biopsy, is reached. If more mobilization of the proximal colon is warranted the inferior mesenteric artery should be divided close to the aorta leaving the marginal vessels intact. Smaller vessels can be divided with monopolar HFE (high frequency electrocautery). Larger vessels can be divided between ligatures or clips, or with ultrasonic energy. They can also be sealed with bipolar HFE and cut with scissors.

5. The rectum once mobilized can be everted transanally and transected outside the body (Figure 5). In this case the everted rectum is amputated close to the anus, after which the aganglionic bowel can be further exteriorized and removed. The distal end of the bowel is then closed and pushed back into the abdomen as is the rectum (Figure 6). The amputated rectum remains open in the abdomen. 


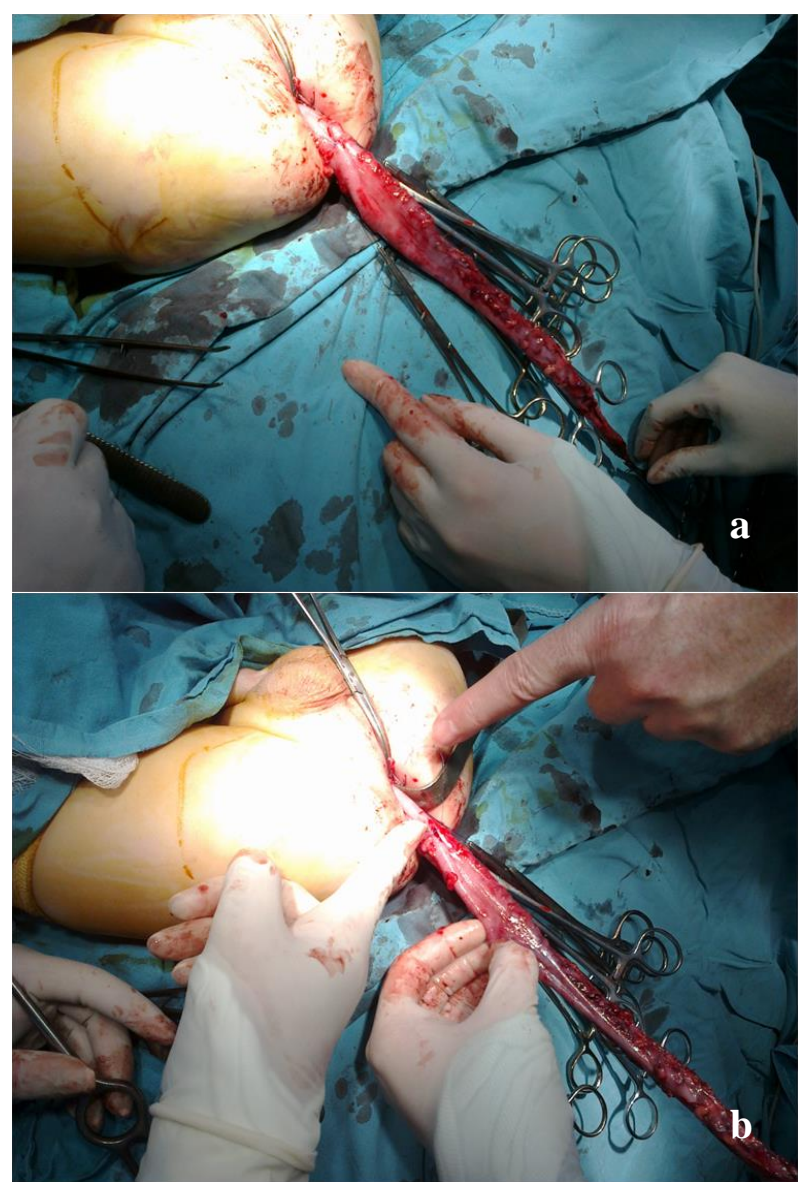

Figure 5(a and b) - Rectum mobilized and everted transanally and transected outside the body

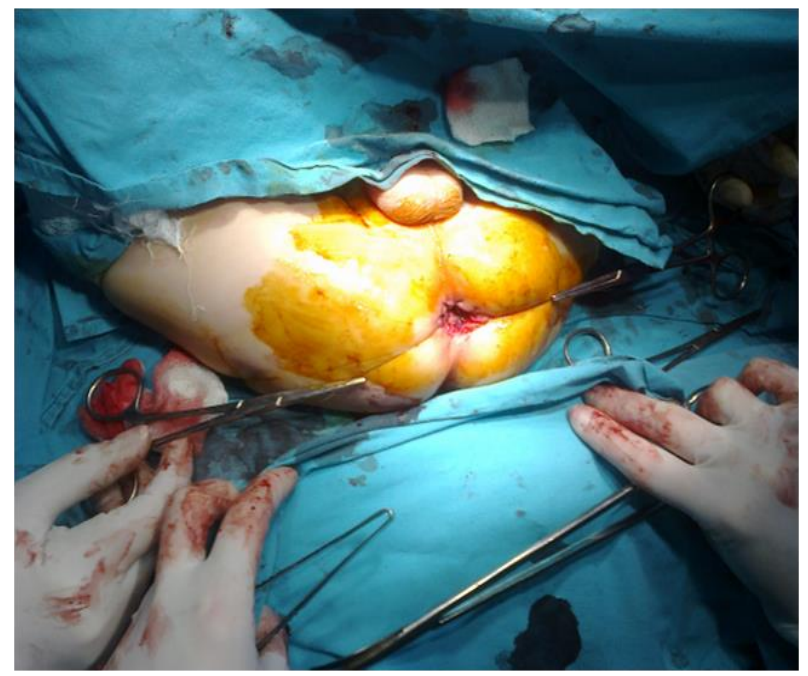

Figure 6 - Distal end of the bowel closed and pushed back into the abdomen as is the rectum

6. Depending on the pacient's age, a transverse incision is made 0.5 to $1 \mathrm{~cm}$ above the dentate line in the posterior rectal wall. The retrorectal space is opened and a curved artery forceps is inserted to grasp the closed end of the mobilized colon under laparoscopic view. The mobilized colon is pulled down through the retrorectal space and opening in the posterior rectal wall until the 'good biopsy site' is reached.
7. Anastomoses: a. Transanal terminolateral: Once the proper colon segment is being reached, it's being retracted through the ,pulltrough" method, it's being amputated and circumferentially anastomosed against the opening of the posterior rectal wall.

b. Transanal latero-lateral: A liniar stapler is being inserted with one $r$ both handles in the ,pulled-through" colon while the other handle is being introduced in the remaining rectum. The laterolateral anastomosis is finalized once the two margins of the plague are air-tight, the rectum being put back into his abdominal position. Finally the rectal blunt end is being sutured laparoscopically.

8. As the rectum is being amputated and pushed back into the abdomen, the open upper portion has to be closed. This is done laparoscopically with a running suture. The needle and suture are inserted directly through the abdominal wall.

\section{Results}

Postoperatively, the patient will receive intravenous fluids and antibiotics; however, nothing may be administered by mouth until passage of flatus or stool signifies return of bowel function

The most frequent postoperative complications include anastomotic leakage and stricture formation, wound infection, intestinal obstruction, pelvic abscess, and re-operation. After intestinal diversion, patients may also develop enterostomal complications [5], such as prolapse, herniation, or stricture. Enterocolitis, chronic obstruction, incontinence and constipation may occur late after surgery for Hirschsprung disease. Rectovesical fistulae have also been reported in the literature [6].

\section{Discussion}

Hirschprung's Disease was described for the first time in 1886 and should be suspected especially in newborns experiencing abdominal distension and constipation [7]. The surgical treatment has many major advantages from a postoperative perspective however some pacients can develop intestinal dysfunctions, 
stenoses, incontinences, diarrhea, constipation or even enterocolitis [8]. Laparoscopy has been massively developed in the last years and taken into consideration even for treating this type of disease especially from the point of view of postoperative recovery, recovery time, postoperative pain as well as cosmetic inconveniences being minimum compared to other surgical procedures. Among other described technical procedures [9], the Swenson procedure is considered to be the most physiological procedure, minimizing transanal technique issues [10]. The laparoscopically modified Duhamel method presents the advantages of a low rectal anastomosis with the prevention of megacolon residual symptoms [11-13].

\section{Neuronal cell transplantation: latest treatment approach for Hirschsprung disease}

Even though it is still considered an experimental approach to our problem, neuronal cell transplantation [14] represents a major asset for the way we'll be tackling the Hirschprung disease issue in the nearby future and we will also be able to manage postoperative colorectal dysfunction. The strategy is more of a cell replacement therapy verified only in mice at this stage, a strategy [15] that might be useful not only in the Hirschprung disease but also in diseases in which enteric neurons are either absent or abnormal. The preliminary work of recent studies supports the notion that intestinal biopsies from the ganglionated proximal intestine of patients with Hirschsprung disease can serve as a source of enteric neurons for autologous cell replacement. However, repopulating an entire aganglionic segment of human colon requires a large number of cells capable of migrating long distances. Scientists have successfully isolated and expanded neuronal stem cells from the intestine of preand post-natal mice in vitro. They have also transplanted these cells into the colon of wildtype mice and shown that they are able to survive, migrate, and differentiate into a functional neuronal network. In order to reproduce a dense neuroglial network within the gut there are currently being explored several approaches some by modifying the neuronal stem cells in vitro by introducing viral vectors expressing Gdnf to promote their proliferation and migration, or co-transplanting them with serotonin, which both in vivo and in vitro has a significant neurogenic effect on these cells.

\section{Conclusions}

Hirschsprung disease is a neonatal condition requiring surgery, which, most commonly, in the recent years has presented a laparoscopic approach.The Duhamel technique is one of the classical procedures adapted for laparoscopic approach. In this case we chose this method for the extended recto-sigmoidian aganglionosis, the patient showing favorable evolution.

\section{References}

[1]http://emedicine.medscape.com/article/178493overview

[2]Aprodu SG, Doina Mihaila, Botez C. et alDiagnosticul si tratamentul megacolonului congenital prin metode clasice- experienta personala, Jurnalul de Chirurgie 2007; 3: 141-45 [3]http://hirschsprungs-disease.com/surgicalprocedures/duhamel-procedure/

[4]Haricharan RN, Georgeson K. Hirschsprung disease. Seminars Pediatr Surg 2008;17:266-75

[5]Puri P, Holschneider AM. Hirschsprung's disease and allied disorders third edition 2008

[6]http://www.ncbi.nlm.nih.gov/pubmed/10945688

[7]Haricharan RN, Georgeson K. Hirschsprung disease. Seminars Pediatr Surg 2008;17:266-75

[8]Bai Y, Chen H, Hao J, Huang Y, et al. Long-term outcome and quality of life after the Swenson procedure for Hirschsprung's disease. J Pediatr Surg 2002;37:639-42

[9]Lacy AM, Delgado S, Castells A, et al. The longterm results of a randomized clinical trial of laparoscopy assisted versus open surgery for colon cancer. Ann Surg. 2008;248:1-7

[10]Mathur MK, Aggarwal SK, Ratan SK, Sinha SK.Laparoscopic-assisted transanal pull-through for Hirschsprung's disease: Comparison between partial and near total laparoscopic mobilization of rectum. $\mathbf{J}$ Indian Assoc Pediatr Surg. 2014 Apr;19(2):70-5. doi: 10.4103/0971-9261.129596.

[11]Kalloo AN, Singh VK, Jagannath SB, et al. Flexible transgastric peritoneoscopy: a novel approach to diagnostic and therapeutic interventions in the peritoneal cavity. Gastrointest Endosc. 2004;60:114-117

[12] Kantsevoy SV, Jagannath SB, Niiyama H, et al. Endoscopic gastrojejunostomy with survival in a porcine model. Gastrointest Endosc. 2005;62:287- 


\section{2}

[13]Merrifield BF, Wagh MS, Thompson CC. Peroral transgastric organ resection: a feasibility study in pigs. Gastrointest Endosc. 2006;63:693-697 [14]http://www.massgeneral.org/children/news/news article. $\operatorname{aspx} ? \mathrm{id}=4342$

[15]Goldstein AM, Hofstra RMW, Burns AJ. Building a brain in the gut: development of the enteric nervous system. Clinical Genetics 2013;83:307-316. 